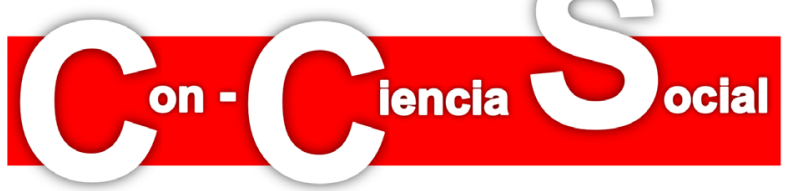

\title{
La administración pública educativa: actuaciones estatales en las dinámicas reformistas y en la determinación curricular en Colombia durante el siglo $\mathrm{XX}$
}

\author{
Public administration in education: State actions in the reform \\ dynamics and in the determination of the curriculum in Colombia \\ during the 20th century
}

\author{
Gina Claudia Velasco Peña \\ Docente Secretaría Educación de Bogotá \\ gorgyas@gmail.com
}

Recibido en diciembre de 2019

Aceptado en enero de 2020

DOI:10.7203/con-cienciasocial.3.16797

\begin{abstract}
RESUMEN
El análisis de los contextos de producción curricular, de los sujetos involucrados y las pugnas desatadas en la definición de los campos de conocimiento y los contenidos culturales que determinan el currículo oficial, pueden mostrar el papel del Estado y de la arquitectura institucional educativa como garantes de la producción y reproducción de unas condiciones sociales específicas para mantener o profundizar la desigualdad sociocultural en el marco del sistema capitalista. Así, las reformas son estrategias o mecanismos estatales para la absorción y adaptación de los lineamientos educativos que se producen en las dinámicas de desarrollo del capitalismo a nivel regional o mundial, mientras que a nivel local, ejerce un rol de regulación y control sobre los procesos educativos por la vía de los campos de conocimiento y contenidos escolares con el propósito de orientar modos de formación destinados a mantener el orden social existente. Estos procesos suelen legitimarse discursivamente con la dialéctica crisisconsenso-fracaso. Este análisis se soporta desde una perspectiva histórica de corte marxista, en la que se teje una argumentación con elementos teóricos sociológicos, jurídicos, politológicos, administrativos y educativos.
\end{abstract}

Palabras clave: Estado, instituciones, educación, reformas, currículo, capitalismo.

\begin{abstract}
The analysis of the contexts of curricular production, of the roles of the subjects involved in the process and the struggles unleashed in the definition of the fields of knowledge and the cultural contents that determine the official curriculum, can show the role of the State and of the educational institutional architecture as guarantors of the production and reproduction of specific social conditions to maintain or deepen socio-cultural inequality within the framework of the capitalist system. Thus, reforms become strategies or mechanisms used by the State for the absorption and adaptation of educational guidelines produced in the development dynamics of capitalism at the regional or global level, while at the local level, it exercises a role of regulation and control over educational processes through the fields of knowledge and school content with the purpose of orienting modes of formation aimed at maintaining the existing social order. These processes tend to be discursively legitimated by the dialectic of crisisconsensus-failure. This analysis is supported from a Marxist historical perspective, in which an argument is woven with theoretical sociological, juridical, political, administrative and educational elements.
\end{abstract}

Keywords: State, institutions, education, reforms, curriculum, capitalism.

\section{Referencia}

Velasco Peña, G. C. (2020). La administración pública educativa: actuaciones estatales en las dinámicas reformistas y en la determinación curricular en Colombia durante el siglo XX. ConCiencia Social (segunda época), 3, 185-196. DOI:10.7203/con-cienciasocial.3.16797 


\section{ESTADO Y EDUCACIÓN: RELACIONES HISTÓRICAS Y TEÓRICAS ${ }^{1}$}

Iniciar un acercamiento a los estudios sobre las reformas educativas, incluidas las curriculares, requiere ubicarlas en el marco de las lógicas y actuaciones del Estado y explicar la manera en que intervienen en la escena de la administración pública como estrategias de absorción y adaptación hacia afuera o de regulación y control hacia dentro. Esta es una tarea compleja, pues son profundos los debates con relación a la historicidad y teorías del Estado que analizan diferentes aristas, tantas, que rebasan el interés particular de este escrito. En tal sentido, el análisis se restringe a retomar aspectos que, desde perspectivas disciplinares diversas permiten explicar teóricamente la relación entre el Estado y el campo educativo, para luego tratar de ubicar el lugar de la institucionalidad en esa materialización vinculada al rol de los sistemas educativos y la escuela.

Como categoría analítica en el contexto de las sociedades capitalistas, el Estado es una esfera política relativamente autónoma, una instancia política que funciona como una dimensión distinta y separada del conjunto social; mientras que su dimensión material "se define por su modo de intervención en relación a la sociedad y a un sistema político" (Vargas Velásquez, 1999, p. 13), en otras palabras, son formas concretas de organización, estructura y actuación de un cuerpo político como resultado de un proceso sociohistórico y cultural particular. Las teorías marxistas expuestas por Bruzón Viltres (2012), relacionan conceptualmente al Estado con un aparato suprasocial cuya función es imponer la voluntad política de la clase o sector hegemónico sobre el resto de la sociedad, reforzando desde el plano ideal, la ilusión del interés común que constituye el contenido cultural de la nación y en el cual basa su poder ideológico. En este caso, la hegemonía se sostiene en la idea del consenso para conferir a los sistemas y formas de organización estatal la autoridad, legitimidad y soberanía para someter e imponer, lo que lleva a considerar la dominación estatal como forma hegemónica de organización del poder social. Se excluye así la idea de neutralidad, pues la administración pública se ubica en el intersticio de estrategias de dominación política, de una arquitectura institucional estatal, de directrices oficiales, intereses de clase y de élites, también, de la influencia de las esferas política y económica.

\footnotetext{
1 Este texto nace de la tesis doctoral, sustentada en marzo de 2019, en el Doctorado Interinstitucional de Educación de la U. Distrital de Bogotá (Colombia) (Velasco, 2019).
} 
A través de las instituciones públicas, entre ellas la escuela, el Estado interviene en la vida social, por ello, la institucionalidad se presenta como una estructura con limitada o relativa autonomía en la que coexisten relaciones orgánicas, de interdependencia, sistémicas o jerárquicas que comparten un núcleo de ideas, valores, principios y objetivos con una modalidad organizativa de subordinación a un órgano de dirección que las congrega, coordina, controla o administra. Estado e instituciones proporcionan base jurídica, unidad y coherencia de forma a la trama social en la que se desenvuelve la vida del hombre común. Es decir, transmiten referentes y pautan los marcos de actuación que confieren identidad y estabilidad al conjunto social; es en este sentido en el que nos interesa interrogar la relación entre las instituciones políticas y las sociales, así como la manera en que se configuran su relaciones para perfilar un orden social determinado y cómo a través de ellas transita y se transmite el poder y la cultura.

Analizando de esta manera las dinámicas de organización social, las instituciones son "una tipificación recíproca de acciones habituales por parte de determinados tipos de actores" (Berger y Luckmann, 2003, p. 74), son un producto histórico tamizado socialmente que funda tradiciones para transmitir a las estructuras y procesos estatales la estabilidad necesaria para sostener y reproducir el sistema en su esencia. Por ejemplo, el funcionamiento del Ministerio de Educación en Colombia funciona sobre la base de tradiciones instaladas en la gestión y administración de la educación difíciles de modificar, incluso sobreviven a reestructuraciones administrativas porque la cultura burocrática constituye una permanencia palpable que supera incluso los cambios de gobierno, de sistemas políticos o de los ajustes institucionales.

Adicionalmente una institución previene cambios no deseados, para lo cual se predefinen mecanismos de ajuste cuando surgen $o$ varían situaciones no contempladas, cuando no se cumplen los objetivos o los resultados no son los esperados, por ello se someten a frecuentes transformaciones para adaptarse a las condiciones variables del medio o para estabilizar la correlación de fuerzas en la distribución desigual del poder. Aquí encuentran nicho las reformas como mecanismos de adaptación hacia afuera y como estrategia de operación interna para ajuste, regulación y control. 


\section{RELACIONES ESTATALES E INSTITUCIONALES EN LA ADMINISTRACIÓN DEL SECTOR EDUCATIVO en Colombia}

Las relaciones entre Estado-escuela-sociedad se sellaron con la creación de una estructura burocrática y administrativa para los asuntos educativos controlada por el Estado. La adjudicación de recursos, tiempos y espacios para el desarrollo de las actividades escolares formalizaron la organización y funcionamiento de la escuela, definieron un marco legal, configuraron roles y rituales específicos y retomaron misiones asignadas socialmente de manera declarada o implícita. La organización de un sistema de administración escolar público se convirtió la forma de intervención política en la esfera social más importante para los Estados liberales y un tópico recurrente en temas de reforma y modernización, porque idealizarían la quimera de igualdad para los ciudadanos, la integración sociocultural y la legitimación política, base de un nuevo imaginario colectivo.

La construcción de una administración estatal educativa en Colombia supuso un gran esfuerzo en términos de financiación, establecer una arquitectura legal para definir la mecánica de organización, articulación, funcionamiento, gestión, dirección y control; asuntos muy difíciles de lograr por las condiciones precarias y de aislamiento en las que se encontraban los establecimientos en los que se impartía la instrucción. En el primer tercio del siglo $\mathrm{XX}$ todavía no se contaba con un sistema educativo en sentido estricto, debido a la inexistencia de una política educativa y la indiferencia hacia el sector, a lo que se suma la disgregación y desarticulación de establecimientos educativos y la dicotomía público-privada, muy acusada especialmente en el nivel de educación secundaria. Fue a mediados de siglo cuando se esboza una organización relativamente estable, pero aún con serias falencias en la administración, tal como se manifiesta en las memorias ministeriales de 1961, en las que se reconoce "la falta de coordinación y, en ciertos casos, la ignorancia por parte del Ministerio de Educación de la labor que en materia de educación y cultura llevan a cabo otras dependencias" lo cual "no permite un balance de realizaciones" (Colombia. Ministerio de Educación Nacional, 1960, p. 12).

Con todo, en el siglo XX, la asignación de nuevas funciones sociales al Estado, produjo una progresiva expansión de la escuela con legislaciones que establecieron la obligatoriedad de la educación básica y la configuración del sistema educativo en cuyo seno se definió una segmentación basada en una escala social y académica, fijando para ello, niveles paralelos diferenciados tanto en los planes de estudio como en el 
origen social de los alumnos. El sistema educativo y la escuela logran así imbuirse en los terrenos de la gobernabilidad, la autoridad, la dominación, el poder y la disciplina.

Podría decirse que la escuela ha estado ligada históricamente a la función estatal de reducir la incertidumbre social y lograr niveles aceptables de estabilidad, es decir, la institución escolar se ve en diferentes momentos y contextos como un mecanismo o instrumento para la emancipación, para superar la desigualdad, para promover la movilidad social, entre otros. Por ejemplo, en los discursos oficiales de presidentes y funcionarios del ramo es frecuente encontrar el argumento del imperativo social y moral que conllevan los procesos escolares para formar generaciones que puedan engrandecer la nación e impulsar los sectores de la economía para generar progreso y desarrollo. Por ejemplo, el presidente Guillermo León Valencia propuso en su campaña de 1962 que las políticas gubernamentales deberían enfocarse en lo social y proyectar su impacto a las mayorías, principalmente a los más pobres, para solventar las necesidades más apremiantes, entre ellas las educativas, "porque solo así podrá hablarse de un verdadero desarrollo armónico y no de un desarrollo superficial y monstruoso que siga acumulando en pocas manos los resultados del común esfuerzo y ahondando las ya existentes desigualdades" (Ayala Diago, 2011, p.96). Los pedagogos coinciden en la importancia y la necesidad imperante de educar a la sociedad, Zapata (1931), por ejemplo, señala que "la educación pública ha de consistir en la igualdad de condiciones y de medios para todos, a fin de que la desigualdad que determina la vida sea originada por la diferencia de facultades y no por prerrogativas de otra especie" (p. 47).

Recapitulando, la educación ha sido presentada como uno de los grandes consensos sociales, un bien supremo mediante el cual es posible cimentar colectivamente un arquetipo sociocultural y un medio para difundir los conocimientos indispensables para lograr la inserción social y ascenso económico de los individuos, reducir las desigualdades y consolidar un determinado proyecto social. Estos elementos son recogidos para posicionar discursiva o ideológicamente a la educación y su función social; así las cosas, la educación es vista como un factor determinante en la construcción de las perspectivas, aspiraciones y roles individuales como sociales. En este imaginario descansa la idea sobre la reforma de la escuela como mecanismo para lograr la reanimación económica, la transformación cultural y la solidaridad nacional (Popkewitz, 1994, p. 25). Esta visión inspiró las políticas educativas en América Latina y 
orientó la distribución de la inversión pública principalmente porque la idea de cambio y mejora impacta con fuerza en la opinión ciudadana.

La relación economía-educación se afianza desde mediados de siglo, con la creencia que la evolución, el progreso, el desarrollo, la modernización o cualquier estado de mejora social descansa en el conocimiento técnico-científico y en su difusión a través de los sistemas de educación, por tanto, el capital cultural deviene en capital económico. Con este sólido argumento, las prescripciones justifican las reformas con necesidades de naturaleza económica, como ocurrió en Colombia con los decretos 1124 de 1948 y 2117 de 1962 que crearon modalidades consideradas claves para el desarrollo material: "el incremento de la enseñanza técnica es indispensable en el país con el fin de intensificar la capacitación especializada de las clases laboriosas, y el mayor desarrollo del aprendizaje de cuestiones prácticas tanto industriales como agrícolas" (Colombia. Presidencia de la República, 1948); también se considera "de la mayor conveniencia" intervenir en enseñanza comercial "para que cumpla con exactitud la función de preparar personal eficiente" para labores administrativas y financieras en las áreas comercial, bancaria, industrial y de la Administración Pública; "y armonizar la capacidad técnica con las condiciones esenciales para la vida cívica, cultural y familiar" (Colombia. Presidencia de la República, 1962).

No obstante, la relación determinista o causal entre escolarización y desarrollo económico no fue la esperada, en principio, porque el sistema educativo en Colombia funciona como un embudo que impide a la mayoría que ingresa a la primaria llegar a estudios medios y superiores, constancia de ello son las cifras oficiales de mediados de siglo: el $60 \%$ de los que ingresan al sistema alcanzan a cursar cuarto año de primaria, un $25 \%$ culmina la secundaria y se titula, pero sólo el $15 \%$, ingresa a la Universidad (Colombia. Ministerio de Educación Nacional, 1942, pp. 43-44). Con todo esto, el optimismo modernizador de alcanzar el progreso por la vía educativa, tuvo su ocaso en los ochenta con el fin de la metáfora del desarrollo y el comienzo del viraje hacia el neoliberalismo; esto desvirtuó las posiciones oficiales sobre la alta movilidad social en Colombia derivada de la escolarización y, como en casos similares, se reconoció la persistencia de la crisis y nuevamente la reforma era la respuesta al fracaso, esto para reiterar la intención de encauzar la acción oficial para superar la pobreza o la desigualdad, pero sin reconocer que no se está dispuesto a alterar la jerarquía social. 


\section{CONSENSOS DE LA ADMINISTRACIÓN EDUCATIVA ESTATAL: CRISIS Y REFORMAS}

Constantemente se alude a crisis de Estado, institucionales, de los modelos económicos o políticos o cualquier situación donde lo disfuncional, desajustado, deficiente y excesivo, constituyen una negación permanente de un orden establecido y conocido, forzando un reordenamiento de las estructuras de relaciones sociales. En suma, la crisis pone en riesgo la legitimidad, equilibrio y estabilidad del sistema, generando repercusiones diferenciadas en la percepción, opinión y sensación de bienestar de los ciudadanos. Por su parte, el consenso reviste de legitimidad el uso y ejercicio del poder, busca la adhesión de la voluntad ciudadana, en la medida que muestra relación y coincidencia entre las aspiraciones políticas del individuo y los objetivos de quienes manejan el poder. De ahí que crisis y consenso sean esenciales en las justificaciones estatales para respaldar la toma de decisiones construye una premisa que proporciona solidez a los discursos con los que se intenta movilizar la sensibilidad y moralidad social, esencia de la opinión pública.

Así, permanentemente instalado en un estado de crisis y caos, el sistema de enseñanza constituye un esencial instrumento de reproducción y legitimación de las condiciones que garantizan la conservación del orden social imperante porque "el ininterrumpido proceso de adaptación - reformas, reformas, reformas- puede considerarse como la mejor garantía de fortalecimiento, tanto de su lógica interna de funcionamiento, como de los efectos que produce en los distintos ámbitos de la estructura social" (Lerena Alesón, 1989, p. 17).

En este sentido, las reformas educativas y curriculares operan como mecanismos de regulación, control, adaptación y ajuste a determinadas situaciones internas, principalmente demandas que provienen de las clases o grupos hegemónicos, o exógenas que requieren adaptarse a las dinámicas económicas internacionales.

Con frecuencia, las memorias de los ministros ubican en el centro del discurso la baja calidad de la educación que deviene en una crisis generalizada que amenaza con escalar, asegurando, además, que es una percepción y necesidad compartida:

aun cuando habla este progreso en el aumento de institutos [...], la marcha de éstos era deficiente por múltiples razones [...] El clamor contra estas cosas era unánime en el país, y ciudadanos y estudiantes anhelaban una reorganización o reajuste en la marcha de los colegios. (Colombia. Ministerio de Educación Nacional, 1946, p. 71) 
es un clamor nacional la insatisfacción de nuestro pueblo con la educación que está recibiendo. (Colombia. Ministerio de Educación Nacional, 1959, p. 6)

El uso de calificativos que enfatizan la gravedad del problema podría explicar la eficacia del arsenal discursivo y algorítmico gubernamental, algunos vocablos abarcadores soportan la idea de consenso y, si no es suficiente, se refuerza con expresiones vehementes o de marcado tinte populista. Todos estos elementos retóricos movilizan la sensibilidad frente a los asuntos públicos y facilitan el trámite de la reforma en el Congreso o la presidencia. Teóricamente, la decisión legislativa adecua las instituciones con base sobre lo que se considera deseable, factible y socialmente aceptable" (Valencia Escamilla, 2012, p. 13), no obstante, la materialidad legislativa es resultado de una racionalidad política en la cual los actores con un poder relativo en una situación coyuntural, entran en conflicto, buscan aliados, negocian, se movilizan e intercambian concesiones y buscan imponer sus intereses.

En cuanto a las dinámicas reformistas en el contexto mundial, se ubican en un entorno inestable con ritmos acelerados y cambiantes, con interjuegos de poder desigual de una alta complejidad. Aquí los actores con mayor poder y voluntad marcan a los recipiendarios los límites de acción basados en unos "principios supremos de reparto" o regímenes que son regulados por organismos internacionales, quienes hacen cumplir lo que generan los repartidores, pero sin capacidad de generación de reglas.

Uno de los rasgos de la política internacional son las densas y asimétricas redes de relaciones de interdependencia o cooperación, que luego se convierten en dependencia por la posición dominante de algunos actores con los que se establecen alianzas o relaciones estratégicas. Esta tendencia ha minado la noción de soberanía logrando que problemáticas, centradas principalmente en temas estructurales de seguridad, economía y política, se tornen en asuntos de competencia regional o global, de ahí los mecanismos de integración y políticas comunes transferidas a las dinámicas internas que, a través de las reformas, se implementan refractariamente.

Hasta mediados de siglo XX, la injerencia internacional en lo educativo no fue tan determinante, las primeras alusiones a la coordinación con la UNESCO se remontan a 1947. Un proceso iniciado en 1955 por solicitud del ministro de Gabriel Betancourt Mejía culmina en 1957 con el posicionamiento del planeamiento en la esfera pública al crear la Oficina de Planeación del Ministerio, dependencia que estudia y proyecta 
planes de trabajo, coordina y evalúa los programas de acción y asesora a las Divisiones de trabajo; con la asistencia técnica de expertos suministrados por la UNESCO y en conjunto con la Misión de Asistencia Técnica del gobierno alemán se elaboró el Informe para el Primer Plan Integral de la Educación que luego se convirtió en el Primer Plan Quinquenal de Educación. El asesoramiento recibido en 1960 incluye equipos que sugieren, de acuerdo con su especialidad, la dirección o reformulación de las políticas públicas educativas, vínculo que lleva a la progresiva desnacionalización en la producción de orientaciones y reformas educativas. En la actualidad, el impulso reformista emerge principalmente de la divergencia con las dinámicas económicas capitalistas regionales o globales, pero para legitimarlas, siguen presentándose como acuerdo para superar la crisis en los consensos sociales locales y promesa de crecimiento económico.

\section{LA DETERMINACIÓN CURRICULAR ENTRE 1939 Y 1974 EN COLOMBIA}

Durante este período, los planes de estudio fueron tópicos recurrentes en la enunciación de los problemas educativos y el consenso la vía por la cual se moviliza discursivamente la declaratoria del estado crítico generalizado y la urgencia de crear contingencias para sosegar las aspiraciones sociales. En las memorias de los ministros, en los informes de funcionarios y en los medios de prensa se expresaba que los principales factores asociados a la crisis eran la financiación; la falta de organización y estructuración del sistema y del ministerio; la calidad de la formación docente; y la obsolescencia e impertinencia de los planes de estudio. En el último asunto, la oficialidad coincide en que los planes de estudio, como vehículos culturales, deben estar en sincronía con los avances científicos y pedagógicos, de tal manera que la obsolescencia de contenidos y métodos es la responsable del rezago social, cultural y económico de la nación. Así mismo, la reforma curricular se vuelve recurrente en la gestión ministerial, pero sin éxito aparente.

Los constantes fracasos eran efecto natural de tener una lánguida estructura institucional, pues el ministerio no contaba con la estructura administrativa ni la fortaleza financiera para solucionar los problemas del sector, mucho menos para construir y desarrollar una propuesta educativa nacional. A esto se suma, que los ministros no tuvieron una estabilidad en la ocupación del cargo, la mayoría no superaba el año o eran asignados en provisionalidad mientras eran titulares en otra cartera. Entre los ministros, aunque tuvieron mayoritariamente experiencias como catedráticos 0 
directivos en universidades, en su mayoría eran "ministros de profesión" que deambularon por diferentes carteras. Las ocho reformas a los planes de estudio que cursaron a lo largo de los treinta y cinco años analizados y los once decretos emitidos son muestra de lo anterior.

Las modificaciones a planes y programas son frecuentes, incluso fugaces, algunas medidas se archivaron o tuvieron una vigencia inferior a un mes. Por supuesto, este vértigo reformista nada tenía que ver con lo que se declaraba en documentos públicos o de prensa sobre la creación de políticas estables o la urgencia de actualizar los contenidos escolares, pues entre 1939 y 1974 la estructura de los planes de estudio permaneció prácticamente invariable, se impusieron los modelos lineales caracterizados por la verticalidad y aislamiento entre sus componentes, su marcado enciclopedismo y la rigidez de sus componentes disciplinares, lo cual conllevó a la inclusión de un gran volumen de materias, algunas pertenecientes a un mismo campo de conocimiento, pero centradas en asuntos muy específicos que impedían acercamientos progresivos o continuidades.

Hasta 1955 los ajustes fueron superficiales y tuvieron un débil impacto ya que se limitaron, en su mayoría, a realizar modificaciones insulsas en la estructura de la secundaria, a reorganizar las asignaturas y su intensidad horaria, pero sin afectar en lo esencial su epistemología. Los planes de estudio, muy tradicionales y de marcada impronta político-ideológica conservadora exhibían un claro diferencial social según la clase, género y etnia de sus destinatarios.

Cambios con mediana profundidad inician entrados los años cincuenta cuando incursiona el concepto de currículo e inicia un tránsito que lleva a la transformación de función y sentido de la educación, esta vez supeditada a una lógica utilitarista, de eficiencia del conocimiento en el marco del sistema productivo. A partir de 1962 se va consolidando una estructura compleja que incluye, además de los contenidos, los fundamentos pedagógicos y la intención formativa. Los decretos emitidos en 1962 y 1974 incorporan los principios de planeamiento y calidad, provocando un giro en la orientación de la política educativa y se alcanza a vislumbrar la desnacionalización en la producción curricular, pues no sólo se promueve desde instancias y organismos internacionales, tuvieron además la intención de incluir las orientaciones, recomendaciones y condicionantes que favorecen intereses supranacionales y transfirieron directamente al sector educativo las demandas del sector económico y productivo, para instalar un nuevo orden educativo con un deliberado propósito 
refundacional, que no es otra cosa que sintonizar los sistemas educativos a las exigencias de la globalización económica en cuanto a estructuras organizativas, administrativas, curriculares y pedagógico-didácticas (Martínez Boom, 2004).

La configuración curricular, en este marco de análisis, demuestra que funciona en un enfoque sistémico dentro de la esfera sociocultural que funge tanto como mecanismo de legitimación y reproducción de los procesos de dominación colonial, jerarquización y división social, como para la exclusión en virtud de condición de clase, etnia y género.

\section{REFERENCIAS}

Ángulo, R., Ezevedo, J. P., Gaviria, A. y Páez, G. N. (2014). Movilidad Social en Colombia. En A. Montenegro y M. Meléndez, Equidad y movilidad social. Diagnósticos y propuestas para la transformación de la sociedad colombiana (pp. 37-63). Bogotá: Universidad de los Andes, Departamento de Planeación Nacional. Ayala Diago, C. A. (2011). La explosión del populismo en Colombia. Anapo y la participación política durante el Frente Nacional. Bogotá: Universidad Nacional de Colombia.

Berger, P. L. y Luckmann, T. (2003). La construcción social de la realidad. Buenos Aires: Amorrotu Editores.

Bruzón Viltres, C. J. (2012). Notas sobre Teoría General del Estado. Estudios teóricos básicos sobre el fenómeno estatal y el sistema político de la sociedad. Editorial Académica española. Recuperado de https://fisyp.org.ar/media/uploads/estado.pdf

Colombia. Ministerio de Educación Nacional. (1946). Memoria del señor Ministro de Educación Nacional al Congreso de 1946. Bogotá: Imprenta Nacional.

Colombia. Ministerio de Educación Nacional. (1948). Memoria del Ministro de Educación, 1948. Bogotá: Prensas del Ministerio de Educación Nacional.

Colombia. Ministerio de Educación Nacional. (1959). Memoria del Ministro de Educación al Congreso de 1959. Abel Naranjo Villegas. Bogotá: Imprenta Nacional. 
Colombia. Ministerio de Educación Nacional. (1960). Memoria del Ministro de Educación al Congreso (Vol. 2). Bogotá: Imprenta Nacional.

Colombia. Presidencia de la República. (1962). Decreto Número 2117 de 1962 (agosto 19)]. Diario Oficial (30889). Bogotá.

Lerena Alesón, C. (1989). Escuela, ideología y clases sociales en España. Crítica de la sociología empirista de la educación. Barcelona: Círculo de Lectores.

Martínez Boom, A. (2004). De la escuela expansiva a la escuela competitiva. dos modos de modernización educativa en América Latina. Bogotá: AnthroposConvenio Andrés Bello.

Popkewitz, T. S. (1994). Sociología política de las reformas educativas. El saber/poder en la enseñanza, la formación del profesorado y la investigación. Barcelona: Ediciones Morata; Fundación Paideia.

Valencia Escamilla, L. (2012). La relación Ejecutivo-Legislativo en la elaboración de políticas públicas. CONfines de relaciones internacionales y ciencia política, 8(16), 11-37. Recuperado de http://www.scielo.org.mx/scielo.php?script=sci_ arttext\&pid=S1870-35692012000200002

Vargas Velásquez, A. (1999). Notas sobre el Estado y las políticas públicas. Bogotá: Almudena Editores.

Velasco Peña, G. C. (2019). Las reformas al currículo oficial: la configuración de las ciencias sociales escolares en la educación secundaria en Colombia (19391974) (Tesis doctoral dirigida por el Dr. Luis Carlos Ortíz). Bogotá: Doctotado Interinstitucional en Educación, Universidad Distrital Francisco José de Caldas. Zapata, R. (1932). La Educación y la instrucción en Colombia. Bogotá: Minerva S.A. 